\title{
THE STRUCTURE OF TWO-PARABOLIC SPACE: PARABOLIC DUST AND ITERATION.
}

\author{
JANE GILMAN
}

\begin{abstract}
A non-elementary Möbius group generated by twoparabolics is determined up to conjugation by one complex parameter and the parameter space has been extensively studied. In this paper, we use the results of [7 to obtain an additional structure for the parameter space, which we term the two-parabolic space. This structure allows us to identify groups that contain additional conjugacy classes of primitive parabolics, which following 14] we call parabolic dust groups, non-free groups off the real axis, and groups that are both parabolic dust and non-free; some of these contain $\mathbb{Z} \times \mathbb{Z}$ subgroups. The structure theorem also attaches additional geometric structure to discrete and non-discrete groups lying in given regions of the parameter space including a new explicit construction of some non-classical T-Schottky groups.
\end{abstract}

\section{INTRODUCTION}

Non-elementary Möbius groups generated by two parabolic transformations are parameterized by a non-zero complex number, $\lambda$. This parameter space has been studied extensively. The study begins with the work of Lyndon and Ullman [11] who obtained results about the free part, those values of $\lambda \in \mathbb{C}$ corresponding to free groups and David Wright who, in the 1970's, obtained computer pictures of the portion of the discrete free part that has come to be known as the Riley slice, $\mathcal{R}$ [14, 18] (see also [5, 16]). Subsequently Keen and Series studied the Riley slice and its boundary [10]. Beardon has extensive results on free and non-free points [4, 3]. Bamberg [2] also studied the non-free points. Gehring, Machlachlan, and Martin [6] have studied points for which $\lambda$ gives an arithmetic group. Recently Agol [1] has classified the non-free discrete groups via the topology of a corresponding knot. The discrete two parabolic groups are, of course, Kleinian groups.

In [7, Gilman and Waterman found explicit equations for the boundary for the subspace consisting of classical T-Schottky groups. The

Date: December 12, 2006 .

Research supported in part by grants from the NSA and the Rutgers Research Council and by Yale University. 
boundary consisted of the portions of two parabolas between their intersection. In addition they found a one complex parameter family of non-classical T-Schottky groups. NSDC groups, classical T-Schottky groups and non-classical T-Schottky groups are all either inside the Riley slice or on its boundary. Groups interior to the Riley slice correspond to discrete groups where there are two, but not three conjugacy classes of maximal parabolic subgroups, that is, quotient surfaces or handlebodies where two, but not three curves have been pinched. In [7] it was shown that only four values of $\lambda$ on the boundary of the classical T-Schottky space correspond to additional pinching. These are $\lambda= \pm i, \pm 2$.

Here we are interested in all groups whether or not they are discrete and we want to identify classes of non-discrete groups by the algebraic properties of $\lambda$. These algebraic properties are strongly related to geometric properties of the action of the group on $\hat{\mathbb{C}}$.

We use the results of [7] to obtain an additional structure theorem for the parameter space, which we term the two-parabolic space. This structure allows us to identify groups that contain additional conjugacy classes of primitive parabolics, which following [14] we call parabolic dust groups, non-free groups off the real axis, and groups that are simultaneously parabolic dust and non-free.

\section{Organization}

The organization of this paper is as follows. Section 3 contains some background, notation and terminology. Some readers may skip or skim this section. The body of the paper begins with section 4 where the relation between $\lambda$ and the matrices of the generators is defined and iteration of $\lambda$ is introduced. Section 5 reviews definitions for Schottky groups and prior results about the classical T-Schottky boundary. The results of these two sections are combined to obtain the idea of $n$th classical T-Schottky groups and the main results about such groups (Theorems 6.2,7.1, 7.3 and 7.6 and corollaries 7.2 and 7.4). Section 8 describes the tessellation of the complex plane by the classical T-Schottky boundary and its pre-images and gives some example. Section 9 introduces a new concept of iteration, lateral iteration and discusses moving around the parameter space using combined lateral and vertical iteration (proposition 9.1). The regions for which we find equations and boundaries correspond to types of configuration of Schottky curves on $\hat{\mathbb{C}}$, that is to the geometry of the action of the generators of $G_{\lambda}$ (section 10). We close with some ideas about generalizing the lateral and vertical moves to arbitrary two-generator groups (section 11). 


\section{Contents}

1. Introduction 1

2. Organization 2

3. Preliminaries: Background, terminology and notation 3

3.1. The sometimes topological picture 4

3.2. Properties 4

3.3. Terminology 4

3.4. Parabolics and pinching 4

4. The matrices and iterating $\lambda$.

5. Schottky definitions and prior results 6

6. Iteration and nth T-Schottky groups 8

7. Parabolic dust and Freeness $\quad 10$

7.1. Analysis of $G_{\lambda}$ for $\lambda=\frac{ \pm 1 \pm i}{2} \quad 11$

7.2. Additional Non-free points 12

7.3. Pre-images of cusps on the boundary of the Riley slice 13

7.4. Questions 13

8. Tessellation of $\mathbb{C}$ by the backwards iterates of the Schottky parabolas

8.1. The pre-image of the Schottky parabolas 14

8.2. Second pre-image of the Schottky parabolas 16

9. Lateral Iteration and Vertical iteration 17

10. The action of $S$ and $T$ on $\hat{\mathbb{C}}$.

11. Towards a more general theory 20

12. Acknowledgements 21

References $\quad 21$

\section{Preliminaries: Background, terminology and notation}

We let $\mathbb{M}$ be the Möbius group, the group of two-by-two nonsingular complex matrices acting as linear transformations on the complex sphere $\hat{\mathbb{C}}$ and by extension on hyperbolic three space. We identify $\mathbb{M}$ with $\operatorname{PSL}(2, \mathbb{C})$.

We are interested in two generator subgroups, $G_{\lambda}$, where $\lambda$ is a nonzero complex number, the parameter, and $G_{\lambda}$ is generated by two parabolic transformations. Although the trace of an element of $P S L(2, \mathbb{C})$ is only determined up to sign, if we require that the pull backs to $S L(2, \mathbb{C})$ of the two generators of $G_{\lambda}$ have positive trace, the trace of every other element of $G_{\lambda}$ is well-defined. We use $\operatorname{Tr}$ to denote the trace of an element of $G_{\lambda}$ or its appropriate pull-back to an element of $S L(2, \mathbb{C})$. A transformation is parabolic, of course, if its trace is 
two. A group is non-elementary if it contains no abelian subgroups of finite index. The precise matrices that generate $G_{\lambda}$ will be given below (section 4).

3.1. The sometimes topological picture. We let $\Omega\left(G_{\lambda}\right)$ denote set of discontinuity of $G_{\lambda}$. The group $G_{\lambda}$ may or may not be discrete, but if it is discrete it can have at most three conjugacy classes of maximal parabolic subgroups. The Riley slice of Schottky space is denoted by $\mathcal{R}$ and is defined to be the set of $\lambda$ where $G_{\lambda}$ is discrete and free and contains exactly two conjugacy classes of primitive parabolics so that $\Omega\left(G_{\lambda}\right) / G_{\lambda}$ is a four punctured sphere. We note that when such a $G_{\lambda}$ is discrete, the corresponding hyperbolic three manifold will be doubly cusped at the images of the parabolic fixed points of the group and the corresponding boundary of the handlebody will be a four punctured sphere where the punctures are identified in pairs, that is, a surface of genus two where two curves have been pinched to a point.

When the group $G_{\lambda}$ is not discrete, there is no topological picture to think of.

3.2. Properties. We will be interested in describing sets of $\lambda$ where $G_{\lambda}$ has a number of different algebraic and/or geometric properties. By abuse of language, we say that $\lambda$ is free meaning that $G_{\lambda}$ is a free group. We say that $\lambda$ is Schottky, T-Schottky, classical T-Schottky, non-classical, non-free, NSDC, etc. if $G_{\lambda}$ is.

3.3. Terminology. If $G$ is any subgroup of $\mathbb{M}$ we call a parabolic element $h \in G$ a primitive parabolic if $h=g^{m}$ for some $g \in G$ and some integer $m$ implies $m= \pm 1$. If $G$ is a Kleinian group acting on $\hat{\mathbb{C}}$ and $p$ is any point fixed by an element of $G$, then $G_{p}$, the stabilizer of $p$ is either a rank 1 or rank 2 parabolic subgroup. A Kleinian group is maximally parabolic if it allows no deformations with more conjugacy classes of parabolic elements.

The group $G_{\lambda}$ might not be discrete and have three or more conjugacy classes of primitive parabolics. Following [14] (page 258) and Wright's 2004 post-card caption, we term a two parabolic generator group, whether it is discrete or not, parabolic dust if it has a third primitive conjugacy class of parabolics. In that case both the group and its parameter $\lambda$ are referred to as dust.

3.4. Parabolics and pinching. If $G$ is a discrete group so that there exists a quotient surface, $S=\Omega(G) / G$ and a quotient manifold, words 
in $G$ correspond curves on $S$. If $G$ has a deformation sending some nonparabolic word to a parabolic, we say that the corresponding curve has been pinched.

The maximal number of elements that can be pinched to parabolics is precisely the number of rank 1 parabolic subgroups that any group isomorphic to $G$ can contain. A group with this largest number of rank 1 parabolic subgroups is maximally parabolic. If $G$ is a Schottky group of rank two, then it can contain at most three rank 1 parabolic subgroups.

\section{The matrices And iterating $\lambda$}

If $G$ is a marked group with two parabolic generators, $S$ and $T$, then up to conjugation $G=G_{\lambda}=\langle S, T\rangle$ where $S=\left(\begin{array}{ll}1 & 0 \\ 1 & 1\end{array}\right)$ and $T=T_{\lambda}=\left(\begin{array}{cc}1 & 2 \lambda \\ 0 & 1\end{array}\right)$ for some nonzero complex number. We assume that $\lambda \neq 0$ so that $G$ is non-elementary.

We note for future reference that since $\operatorname{Tr} \quad S=2, \operatorname{Tr} T=2$ and $\lambda \neq 0$, letting $[S, T]$ denote the multiplicative commutator, we have

$$
\begin{gathered}
\operatorname{Tr}[S, T]-2=\operatorname{Tr} S T S^{-1} T^{-1}-2=4 \lambda^{2} ; \\
\operatorname{Tr} S T^{-1}=\operatorname{Tr} T S^{-1}=2-2 \lambda \\
\text { and } \operatorname{Tr} S T=\operatorname{Tr}(T S)^{-1}=2+2 \lambda . \\
\operatorname{Tr} S T= \pm 2 \Leftrightarrow \lambda=-2, \operatorname{Tr} S T^{-1}= \pm 2 \Leftrightarrow \lambda=2, \\
\operatorname{Tr}[S, T]= \pm 2 \Leftrightarrow \lambda= \pm i .
\end{gathered}
$$

Jørgensen's inequality [?] tells us that $G_{\lambda}$ is not discrete if $|\lambda|<1 / 2$. We refer to the circle $|\lambda|<\frac{1}{2}$ as the Jørgensen circle.

We can also calculate that

$$
T_{\lambda} S T_{\lambda}^{-1}=\left(\begin{array}{cc}
1+2 \lambda & -4(\lambda)^{2} \\
1 & 1-2 \lambda
\end{array}\right)
$$

The matrices $S$ and $T_{\lambda} S T_{\lambda}^{-1}$ generate a subgroup of $G$. We can conjugate $S$ and $T_{\lambda} S T_{\lambda}^{-1}$ by the matrix

$$
A=\left(\begin{array}{cc}
1 & 0 \\
\frac{-1}{2 \lambda} & 1
\end{array}\right)
$$


so that the ordered pair of parabolics $\left(S, T_{\lambda} S T_{\lambda}^{-1}\right)$ is conjugate to the ordered pair $\left(S, T_{\tilde{\lambda}}\right)$ where $\tilde{\lambda}=-2 \lambda^{2}$. Thus $\left\langle S, T_{\lambda} S T_{\lambda}^{-1}\right\rangle \simeq G_{\tilde{\lambda}}$. Here $\simeq$ denotes group isomorphism. This defines $\tilde{\lambda}$ for any $\lambda$.

Definition 4.1. If $\lambda \in \mathbb{C}$, we define $\tilde{\lambda}$ by $\tilde{\lambda}=-2 \lambda^{2}$.

Notation 4.2. We write $G_{\lambda} \supset \simeq G_{\tilde{\lambda}}$ to indicate that $G_{\lambda}$ has a subgroup isomorphic to $G_{\tilde{\lambda}}$. Similarly we write $G_{\tilde{\lambda}} \subset \simeq G_{\lambda}$. In drawing diagrams with a lattice of subgroups, we use the standard notation

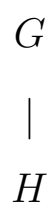

when $H$ is a subgroup of $G$. If $G$ contains a subgroup isomorphic to $H$, we will write

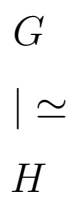

We let $f(z)=-2 z^{2}$. We will obtain information about the structure of two-parabolic space by iterating $f$, a standard procedure in the study of discrete group. Here, we iterate the classical T-Schottky boundary and find potential parabolic dust as well as non-free points.

\section{SchottKy Definitions AND PRIOR RESUlts}

We are interested in T-Schottky groups, which are the same as Schottky groups except that arbitrary tangencies are allowed among the Schottky circles and Jordan curves. That is, tangencies at points that are parabolic fixed points and at non-parabolic fixed points and between circles that are paired or not paired by the marked Schottky generators are allowed.

The picture of the Riley slice is well known. The Jørgensen circle and the classical T-Schottky parabolas are depicted in figure 1. Also depicted is a region in the plane where all group are non-classical TSchottky groups. Groups in the this region which is denoted by $\mathcal{N C F}$ form a one complex parameter family and are called the non-classical family. There are, of course, non-classical groups outside this family. Theorem 5.2 summarizes facts about these regions and their boundaries. In this paper we iterate and apply this theorem to two-parabolic space. First we define 


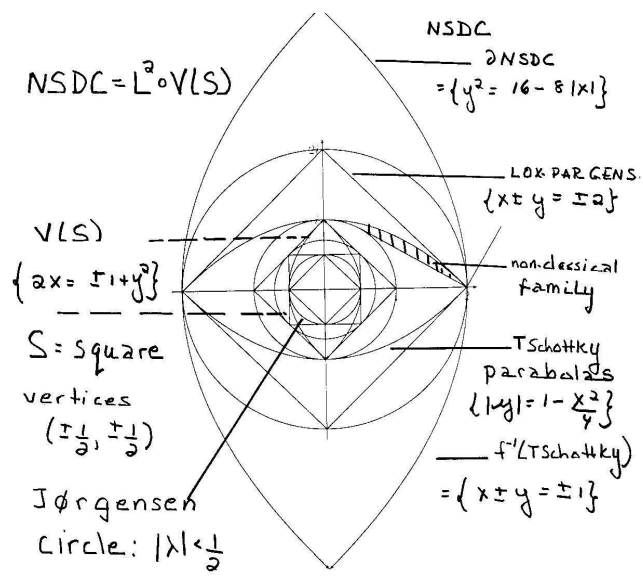

Figure 1. Boundary Regions Each point $\lambda \in \mathbb{C}$ corresponds to a two-generator group. The shaded region shows $\mathcal{N C \mathcal { F }}$ the one parameter family of non-classical T-Schottky groups in the first quadrant. The exterior to the outer parabolas are the non-separating disjoint circle groups (NSDC groups), the middle parabolas are the boundary of the classical groups. The boundary of the Riley slice includes \pm 2 and $\pm i$ but otherwise lies interior to the Schottky parabolas. Points inside the Jørgensen circle $\left(|\lambda|<\frac{1}{2}\right)$ are non-discrete groups. Between the Riley slice and the Jørgensen circle are additional nonclassical groups together with degenerate groups, isolated discrete groups and non-discrete groups.

Definition 5.1. Let $\mathbf{P}=\left\{z=x+i y \mid-2 \leq x \leq 2\right.$ and $\left.\pm y=1-\frac{x^{2}}{4}\right\}$. $\mathbf{P}$ consists of portions of each of two intersecting parabolas. We refer to $\mathbf{P}$ as the as the Schottky parabolas and each portion as a Schottky parabola.

Theorem 5.2. [7] Let $G=\langle A, B\rangle$ with $\operatorname{Tr} A=2$ and $\operatorname{Tr} B=2$ and $\operatorname{Tr}[A, B]-2=4 \lambda^{2}$ where $\lambda \in \mathbb{C}-\{0\}$. Then

(1) $G$ is discrete and classical T-Schottky $\Leftrightarrow \lambda=x+i y$ and $|y| \geq 1-\frac{x^{2}}{4}$.

(2) $\lambda$ is on the boundary of classical T-Schottky space precisely when $\lambda=x+i y,-2 \leq x \leq 2$, and $|y|=1-\frac{x^{2}}{4}$. That is, the Schottky parabolas are the boundary of classical T-Schottky space.

(3) If $G$ is discrete and classical T-Schottky, it has a third conjugacy class of parabolics $\Leftrightarrow \lambda= \pm 2$ or $\pm i$. In the first case, $A^{ \pm 1} B$ is parabolic and in the second case $[A, B]$ is parabolic. 
(4) Let $K$ be the convex hull of the set consisting of the circle $|z|=1$ and the points $z= \pm 2$. If $\lambda$ lies between one of the T-Schottky parabolas and the interior of $K$, then $G_{\lambda}$ is non-classical $T$ Schottky.

Let $L_{K}$ be the line in $\mathbb{C}$ whose equation is $3 y=-\sqrt{3} x+2 \sqrt{3}$. Then $L_{K}$ is tangent to the circle of radius 1 at the point $\left(\frac{1}{2}, \frac{\sqrt{3}}{2}\right)$. Let $K_{0}$ denote the segment between the point of tangency and $z=2$ (including the end points) and let $C_{K_{0}}$ be the arc of the unit circle between the point $\left(\frac{1}{2}, \frac{\sqrt{3}}{2}\right)$ and $i$. Set $B_{K_{0}}=K_{0} \cup C_{K_{0}}$. The the region between the interior of $K$ and the T-Schottky parabola in the first quadrant is the region bounded by $B_{K_{0}}$ and $|y|=1-\frac{x^{2}}{4}$. We denote it together with $B_{K_{0}}$ and its symmetric images across the real and imaginary axes by $\mathcal{N C F}$ and call it the region of the non-classical family. We include in $\mathcal{N C F}$ the line segment $K_{0}$ and the segment of the unit circle between $\pi / 3$ and $\pi / 2$ as well as their symmetric images about the real and imaginary axes. Figure 1 shows the portion of $\mathcal{N C F}$ inside the first quadrant.

Letting $\lambda=x+i y=r e^{i \theta}$ where $r \geq 0$ and $0 \leq \theta<2 \pi$, we obtain an equivalent formulation of 4 .

Corollary 5.3. Let $\lambda=x+i y=r e^{i \theta}$ lie in the first quadrant with $|y|<1-\frac{x^{2}}{4}$. Then $\lambda$ is non-classical T-Schottky if either

(1) $r \geq 1$ and $\frac{\pi}{3} \leq \theta \leq \frac{\pi}{2}$ or

(2) $-\frac{\sqrt{3} x}{3}+\frac{2 \sqrt{3}}{3} \leq y$ and $0 \leq \theta \leq \frac{\pi}{3}$

If $\lambda$ is the image of a point in either of the above two regions under reflection in the $x$ or $y$ axis, then $\lambda$ is non-classical T-Schottky.

Let $\mathcal{A}_{1}$ is the region in corollary $5.3(1)$ and $\mathcal{A}_{2}$ the region in $5.3(2)$. We have by symmetry the regions $-\mathcal{A}_{i}, \overline{\mathcal{A}}_{i}$, and $-\bar{A}_{i}$. Then $\mathcal{N C \mathcal { F }}=$ $\cup_{i=, 1,2}\left(\mathcal{A}_{i} \cup-\mathcal{A}_{i} \cup \bar{A}_{i} \cup-\bar{A}_{i}\right)$.

\section{Iteration AND NTH T-SchotTKy GROUPS}

We are interested in what happens between the Jørgensen circle and the classical T-Schottky parabola. We can iterate as follows. Given $\lambda$ with $\frac{1}{2}<|\lambda|<1, G_{\lambda}$ has a subgroup isomorphic to $G_{\tilde{\lambda}}$ where $\tilde{\lambda}=-2 \lambda^{2}$. We say that $G_{\lambda}$ is second classical T-Schottky if $G_{\tilde{\lambda}}$ is classical TSchottky, but $G_{\lambda}$ is not. We let $f^{n}(z)$ denote the nth iterate of $f$. 
We consider the iterative sequence of subgroups,

$$
\begin{gathered}
\left\langle S, T S T^{-1}\right\rangle \\
\mid \\
\left\langle S, T S T^{-1} \cdot S \cdot\left(T S T^{-1}\right)^{-1}\right\rangle \\
\mid \\
\vdots \\
\mid \\
\left\langle S, T S T^{-1} \cdot S\left(T S T^{-1}\right)^{-1} \cdot S \cdot\left(T S T^{-1} \cdot S\left(T S T^{-1}\right)^{-1}\right)^{-1}\right\rangle
\end{gathered}
$$

That is, we define inductively, the parabolic transformations

$$
\begin{gathered}
P_{0}=T, \\
P_{1}=T S T^{-1}, \\
P_{2}=P_{1} S P_{1}^{-1}, \\
\vdots \\
P_{i}=P_{i-1} S P_{i-1}^{-1} .
\end{gathered}
$$

Set $G_{0}=G_{\lambda}=\left\langle S, T_{\lambda}\right.$ and $G_{i}=\left\langle S, P_{i-1}\right\rangle$. Let $\lambda_{i}$ be the parameter with $G_{i} \simeq G_{\lambda_{i}}$. We have $f^{i}(\lambda)=\lambda_{i}$. Note that as we move down in the lattice of subgroups, $\lambda$ moves out from the origin in $\mathbb{C}$. We define

Definition 6.1. If for some integer $n \geq 1, G_{f^{n}(\lambda)}$ is classical T-Schottky but $G_{f^{(n-1)}(\lambda)}$ is not, we say $G_{\lambda}$ is $n$th classical T-Schottky.

Theorem 6.2. Let $f(\lambda)=\tilde{\lambda}$ and $(f \circ f)(\lambda)=\tilde{\tilde{\lambda}}$. Then

(1) If $|\lambda|<\frac{1}{2}$, then $\left|f^{n}(\lambda)\right|<\frac{1}{2}$ for all integers $n$ so that $G_{\tilde{\lambda}}$ is non discrete and $G_{f^{n}(\lambda)}$ is neither discrete nor classical T-Schottky for any $n>0$.

(2) If $|\lambda|>\frac{\sqrt{2}}{2}$, then $|\tilde{\lambda}|>1$ and $|\tilde{\tilde{\lambda}}|>2$ so that $G_{\tilde{\tilde{\lambda}}}$ is classical T-Schottky.

(3) Given $\frac{1}{2}<|\lambda|<\frac{\sqrt{2}}{2}$, there is a smallest integer $n$ such that if $f(z)=-2 z^{2}$ and $f^{n}(z)$ denotes the $n$th iterate of $f$, then $G_{f^{n}(\lambda)}$ is classical T-Schottky but $G_{f^{(n-1)}(\lambda)}$ is not.

(4) For each $\lambda$ with $\frac{1}{2}<|\lambda|<\frac{\sqrt{2}}{2}$, there is a unique integer $n$ such that $G_{\lambda}$ is nth classical T-Schottky. 
Proof. The first two parts are a calculation using theorem 5.2 which says that if $\lambda \geq 2, G_{\lambda}$ is T-Schottky. To see the third and fourth part for each integer $n$ with $n \geq-1$, define

$$
t_{n}=\frac{1}{2^{\frac{2^{n}-1}{2^{n}}}} .
$$

We consider $C_{r}$, the circle centered at the origin with radius $r$ where $r=t_{n}$. Note that $C_{-1}$ is the circle of radius 2 and $C_{0}$ is the circle of radius 1 . We observe that $\left|f\left(t_{n}\right)\right|=t_{n-1}$ so that $f\left(C_{n}\right)=C_{n-1}$. Since the Schottky parabola lies between the circle of radius 1 and the circle of radius 2, the successive inverse images of the T-Schottky parabolas lie between these successive circles. These circles tessellate the region of the complex plane outside the Jørgensen circle and inside the circle of radius two as do the successive backwards iterates of the Schottky parabolas.

\section{Parabolic dust and Freeness}

We now investigate the pre-images of groups that are either parabolic dust or non-free.

Theorem 7.1. (1) Let $G_{\lambda}$ be such that $f^{n}(\lambda)$ is parabolic dust for some integer $n$, then either $G_{\lambda}$ is parabolic dust or non-free.

(2) Let $G_{\lambda}$ be such that $f^{n}(\lambda)$ is non-free for some integer $n$, then $G_{\lambda}$ is non-free.

Proof. We consider the iterative sequence of subgroups $G_{i} \simeq G_{f^{i}(\lambda)}$ (6.1) and parabolics $P_{i}$ (6.2) and note that as parabolic dust $G_{f^{n}(\lambda)}$ will have three distinct conjugacy classes of parabolic subgroups. Either the generators of these groups are not conjugate in $G_{\lambda}$, in which case $G_{\lambda}$ is parabolic dust or two or more of them are conjugate in $G_{\lambda}$ in which case $G_{\lambda}$ has a relation and is, therefore, non-free. As a non-free group, $G_{f^{n}(\lambda)}$ will have a non-trivial relation and this relation will hold in $G_{\lambda}$.

An immediate corollary is

Corollary 7.2. Let $G_{\lambda}$ be such that $f^{n}(\lambda)= \pm i$ or \pm 2 for some integer $n \geq 1$ then either $G_{\lambda}$ is parabolic dust or non-free.

We will prove that such groups are actually both parabolic dust and non-free. 
7.1. Analysis of $G_{\lambda}$ for $\lambda=\frac{ \pm 1 \pm i}{2}$. We do an analysis of $G_{\frac{ \pm 1 \pm i}{2}}$. Note that $G_{\tilde{\lambda}} \simeq G_{ \pm i}$ and $G_{\tilde{\tilde{\lambda}}} \simeq G_{ \pm 2}$. Here it is important to distinguish between subgroups that are isomorphic to a given group and actual subgroups, that is between $\subset \simeq$ and $\subset$.

Theorem 7.3. $G_{\frac{ \pm 1 \pm i}{2}}$ is both parabolic dust and non-free. It contains two conjugacy classes of $\mathbb{Z} \times \mathbb{Z}$ parabolic subgroups and thus is not free. It contains four distinct conjugacy classes of parabolic elements and thus is parabolic dust.

Proof. By symmetry, it suffices to treat the case $\lambda=\frac{1-i}{2}$.

(1) $\lambda=\frac{1-i}{2}, G_{\lambda}=\left\langle S, T_{\lambda}\right\rangle, T_{\lambda}$ fixes $\infty$ and $S$ fixes 0.

(2) $f(\lambda)=-2 \lambda^{2}=i=\tilde{\lambda}, G_{\tilde{\lambda}} \simeq\left\langle S, T_{\lambda} S T_{\lambda}^{-1}\right\rangle$

Let $\tilde{T}=T_{\lambda} S T_{\lambda}^{-1}$. $\tilde{T}$ fixes $1-i$ and $S$ fixes 0 .

We note that $S \tilde{T} S^{-1} \tilde{T}^{-1}=\left(\begin{array}{ll}3 & -4 \\ 4 & -5\end{array}\right)$ is parabolic with fixed point at 1 .

(3) $\tilde{\tilde{\lambda}}=2, G_{\tilde{\lambda}}=G_{2}$ and $\tilde{\tilde{T}}=\tilde{T} S \tilde{T}^{-1}=\left(\begin{array}{ll}-1 & 4 \\ -1 & 3\end{array}\right)$ has its fixed point at 2 .

(4) We look at $S^{-1} \tilde{\tilde{T}}=\left(\begin{array}{cc}-1 & 4 \\ 0 & -1\end{array}\right)$. Its fixed point is at $\infty$.

(5) Thus $T_{\lambda}$ and $S^{-1} \tilde{\tilde{T}}$ are parabolics that share the same fixed point, but are not conjugate in the Möbius group. Therefore, they must commute

$$
T_{\lambda} S^{-1} \tilde{\tilde{T}}=S^{-1} \tilde{\tilde{T}} T_{\lambda} .
$$

and, therefore, $G_{\frac{1-i}{2}}$ is not free and contains the $\mathbb{Z} \times \mathbb{Z}$ subgroup generated by the commuting elements.

We note that a similar analysis interchanging $S$ and $T_{\lambda}$ shows that $S$ and $T_{\lambda}^{-1}\left(S T_{\lambda}^{-1} S^{-1} T_{\lambda} \cdot\left(S T_{\lambda} S^{-1}\right)^{-1}\right.$ share a fixed point, namely 0 . Since $G_{\lambda}$ is non-elementary, $S$ and $T_{\lambda}$ are not conjugate, none of the four parabolics can be conjugate.

Looking at further pre-images of $\pm i$, we have

Corollary 7.4. If $f^{n}(\lambda)= \pm i$ for some $n>1$, then $G_{\lambda}$ is simultaneously non-free and parabolic dust and contains at least two distinct $\mathbb{Z} \times \mathbb{Z}$ subgroups.

Proof. If $f^{n}(\lambda)= \pm i$, then $f^{(n-1)}(\lambda)=\frac{ \pm 1 \pm i}{2}$ so that $G_{\lambda} \supset \simeq G_{\frac{ \pm 1 \pm i}{2}}$ and inherits its two non-conjugate $\mathbb{Z} \times \mathbb{Z}$ subgroups. 


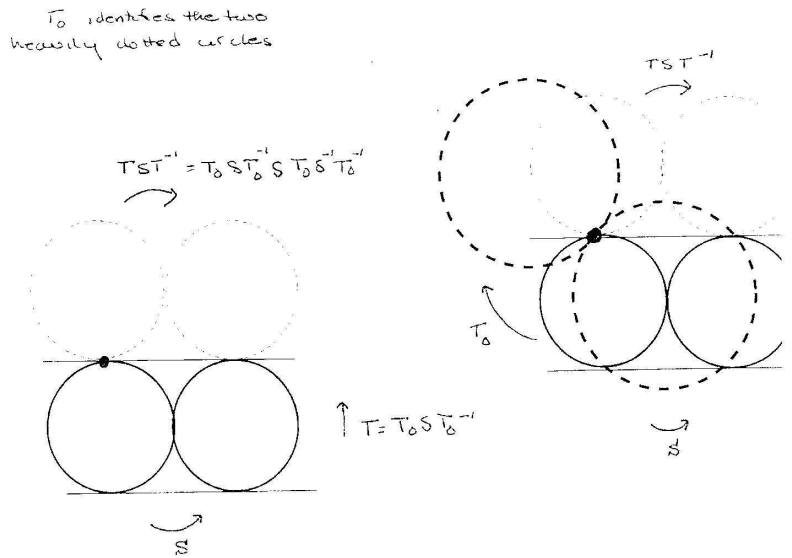

Figure 2. We have conjugated the group $G_{\frac{1-i}{2}}$ so that $S$ identifies $C=\{|z+1|=1\}$ and $C^{\prime}=\{|z-1|=1, T$ identifies $L=\{y=-i\}$ and $L^{\prime}=\{y=i\}, S(z)=\frac{z}{z+1}$, $T(z)=z+2 i, T S T^{-1}(z)=\frac{(1+2 i) z+4}{z-2 i+1}, T_{0}(z)=\frac{2 z+1-i}{\frac{-1+i}{2} z}$, $T=T_{0} S T_{0}^{-1}, G_{\frac{-1+i}{2}} \simeq\left\langle S, T_{0}\right\rangle \supset G_{i}=\langle S, T\rangle \supset G_{2}=$ $\left\langle S, T S T^{-1}\right\rangle$ Then $\stackrel{2}{T}_{0}$ has the same fixed point as $\left[S^{-1}, T\right]$, namely $-(1+i)$.

One could further analyze the pattern of $\mathbb{Z} \times \mathbb{Z}$ subgroups in the pre-images of $\pm i$. We expect the number of non-conjugate such groups to grow.

Figure 2 illustrates the action of (a conjugate of) $G_{\frac{1-i}{2}}$ and its subgroups in $\hat{\mathbb{C}}$, showing the parabolic fixed points and the isometric circles of the transformations. We have conjugated so that $\infty$ is no longer a parabolic fixed point.

Remark 7.5. The $G_{\frac{ \pm 1 \pm i}{2}}$ correspond to the Whitehead link and have finite volume quotients. Since at each step the groups are of infinite index, the corresponding covering would have infinite degree, so the $G_{\lambda}$ with $f^{k}(\lambda)=\frac{ \pm 1 \pm i}{2}$ will have zero volume. That is, the limit sets will be $\hat{\mathbb{C}}$.

7.2. Additional Non-free points. We note that Beardon, Bamberg, Ignatov, and Lyndon-Ullman [3, 4, 2, 8, 9, 11] among others have studied non-free points on the real axis. We combine their results along with theorem 7.1 to obtain complex non-free points. 
Theorem 7.6. Let $\delta \neq 0 \in \mathbb{C}$. Then $G_{\delta}$ is not free if for some integer $k \geq 0 f^{k}(\delta)=\lambda$ and one of the following holds:

(1) $\lambda \in\left\{\frac{p^{2}}{2\left(p^{2}+1\right)^{2}} \mid p=1,2, \ldots\right\} \cup\left\{\frac{1}{2 p^{2}} \mid p=2, \ldots\right\}$

(2) $\lambda=4 \sin ^{2}\left(\frac{p \pi}{q}\right)$ where $p$ and $q$ are relatively prime integers.

(3) $\lambda=\frac{p^{2}}{2 q^{2}}$ where $p$ and $q$ satisfy Pell's equation, $1=q^{2}-N p^{2}$, for some positive square free integer $N$.

(4) $\lambda=\frac{p_{n}^{2}}{2 q_{n}^{2}}, \lambda$ where $N$ is a positive square free integer and the convergents of $\frac{1}{\sqrt{N}}$ are $p_{n} / q_{n}$.

(5) $\lambda \in \mathcal{B}$ where

$\mathcal{B}=\left\{\frac{1}{2}, 1, \frac{3}{2}, \frac{1}{2} e^{\frac{i \pi}{3}}, \frac{1+\sqrt{13}}{4}, \frac{5+\sqrt{5}}{4}, \frac{i}{\sqrt{2}}, \frac{1}{\sqrt{2}}, \frac{9}{50}, \frac{8}{25}, \frac{25}{72}, \frac{8}{81}, \frac{25}{162}, \frac{25}{98}, \frac{9}{8}, \frac{8}{9}, \frac{25}{32}, \frac{25}{18}\right\}$.

(6) $\lambda \in \frac{\lambda_{0}}{n^{2}}$ where $G_{\lambda_{0}}$ is not free.

(7) $\lambda \in\left\{\frac{1}{2}\left(\frac{1}{n}\right)^{2}, \frac{1}{2}\left(\frac{2}{n}\right)^{2}, \frac{1}{2}\left(\frac{3}{n}\right)^{2}, \ldots, \frac{1}{2}\left(\frac{8}{n}\right)^{2}, \quad n \neq 0 \in \mathbb{Z}\right\}$

(8) $\lambda \in\left\{\frac{(m+n)^{2}}{2 m^{2} n^{2}} \mid m \neq 0, n \neq 0 \in \mathbb{Z}\right\}$

Proof. Apply theorem 7.1 to the results of Bamberg, Beardon, Ignatov and Lyndon-Ullman. Translate from the $\mu$ parameter to the $\lambda$ parameter via $2 \lambda=\mu^{2}$ where necessary.

7.3. Pre-images of cusps on the boundary of the Riley slice. In [10] and [17, 18] a family of words $W_{p / q}$ indexed by the rationals, $p / q$, are studied. These are termed Farey words. For a discrete group, words in the group correspond to curves on the corresponding surface. In particular pinching the curves corresponding to Farey words until $\operatorname{Tr} W_{p / q}=-2$ gives cusps on the boundary of the Riley slice, that is, parameters where there are three parabolics. We let $\lambda_{p / q}$ be the value of $\lambda$ for which $\operatorname{Tr} W_{p / q}=-2$. Apply theorem 7.1 to see that

Corollary 7.7. Let $G_{\lambda}$ be such that $f^{n}(\lambda)=\lambda_{p / q}$ for some integer $n$ and some rational number $p / q$. Then either $G_{\lambda}$ is parabolic dust or non-free.

7.4. Questions. Ian Agol has asked the question about the values of $\left|\lambda_{p} / q\right|$. David Wright has computed $|p / q|$ for some values of $p / q$. It would be nice to understand where these points lie in the in the tessellation of the space by the circles $C_{t_{n}}$ that arose in the proof of theorem 6.2.

Can we decide whether the group has a third parabolic conjugacy class or whether it is non-free? Bamberg [2] has a procedure for determining whether $\lambda$ represents a non-free group. It is not an algorithm, that is, it does not necessarily stop, but if one has a bound on the 
length of the word, which we do, and a bound on the exponents of the word in the original generators, his procedure may be useful.

\section{Tessellation of $\mathbb{C}$ By the BaCkWARds iterates of the SCHOTTKY PARABOLAS}

With some more terminology we can say a number of useful things about the backwards iterates of the Schottky parabolas and other boundaries and regions.

Definition 8.1. Let $D_{\diamond}$ be the Euclidean square in $\hat{\mathbb{C}}$ with vertices $\pm i$ and \pm 1 .

8.1. The pre-image of the Schottky parabolas. To investigate the pre-images of $\mathbf{P}$, we first look at what $f$ does to lines. We will see that the function $f$ maps horizontal and vertical lines to parabolas with vertices on the $x$-axis. It also maps all other straight lines to parabolas.

8.1.1. Images of lines. We want to investigate the image of the straight line $x+y=c$ under the map $f(z)=-2 z^{2}$ where $c$ is a real number and $z=x+i y$. We set $f(z)=\tilde{z}=\tilde{x}+i \tilde{y}$.

Lemma 8.2. The image of the line $x+y=c$ under $f$, is the parabola $\tilde{y}=-c^{2}+\frac{(\tilde{x})^{2}}{4 c^{2}}$.

Proof. If $f(z)=\tilde{z}=\tilde{x}+i \tilde{y}, \tilde{x}=-2\left(x^{2}-y^{2}\right)$ and $\tilde{y}=-4 x y$. Calculate that if $x+y=c, y=c-x$,

$$
\begin{gathered}
y^{2}=c^{2}-2 c x+x^{2} \\
x^{2}-y^{2}=2 c x-c^{2} \\
\tilde{x}=-2\left(x^{2}-y^{2}\right)=2 c^{2}-4 c x \\
(\tilde{x})^{2}=4 c^{4}-16 c^{x}+16 c^{2} x^{2}
\end{gathered}
$$

and

$$
\frac{(\tilde{x})^{2}}{4}=c^{4}-4 c^{3} x+4 c^{2} x^{2} .
$$

Also calculate that

$$
\begin{gathered}
\tilde{y}=-4 x y=-4 x(c-x)=-4 x c+4 x^{2} \\
c^{2} \cdot \tilde{y}=-4 x c^{3}+4 x^{2} c^{2} \\
c^{2} \cdot \tilde{y}+c^{4}=c^{4}-4 x c^{3}+4 x^{2} c^{2}=\frac{(\tilde{x})^{2}}{4}
\end{gathered}
$$




$$
\begin{gathered}
c^{2} \cdot \tilde{y}=-c^{4}+\frac{(\tilde{x})^{2}}{4} \\
\tilde{y}=-c^{2}+\frac{(\tilde{x})^{2}}{4 c^{2}}
\end{gathered}
$$

Whence,

Corollary 8.3. If $c= \pm 1, \tilde{y}=-1+\frac{(\tilde{x})^{2}}{4}$.

Corollary 8.4. The diamond, $D_{\diamond}$, is the preimage of the Schottky parabolas.

Proof. Use corollary 8.3 A similar calculation to the one done in proving lemma 8.2 shows that if $x-y= \pm 1, \tilde{y}=1-\frac{(\tilde{x})^{2}}{4}$.

Similar calculations yield

Lemma 8.5. The line $x_{0}=c$ is mapped to the parabola $x=-2 c^{2}+\frac{y^{2}}{8 c^{2}}$.

The line $y_{0}=c$ is mapped to the parabola $x=2 c^{2}-\frac{y^{2}}{8 c^{2}}$.

In particular,

Corollary 8.6. The function $f$ maps the square with vertices at $( \pm c, \pm c)$ to the parabolas $\tilde{x}= \pm 2 c^{2}+\frac{\mp \tilde{y}^{2}}{8 c^{2}}$

and

Corollary 8.7. The function $f$ maps the square with vertices at $\left( \pm \frac{1}{2}, \pm \frac{1}{2}\right)$ to the region bounded by the parabolas $\tilde{x}=\frac{ \pm 1}{2}+\frac{\mp 1}{2}(\tilde{y})^{2}$.

8.1.2. Pre-images of lines. In order to identify the images and preimages of further regions such as $\mathcal{N C F}$ under iteration, we consider preimages of the line $y=m x+b$ where $m \neq 0$. In looking at the regions, we will usually only be interested in segments of lines or parabolas or hyperbolas as we were when we identified the Schottky parabolas as segments of two intersecting parabolas. We adopt the convention the convention that when we say the preimage or image is a line or a parabola or a branch of a hyperbola, we mean the appropriate segment which should be clear from the context.

Lemma 8.8. Let $L=\{z=x+i y \mid y=m x+b\}$ be a straight line with $m \neq 0$ and $b \neq 0$, then $f^{-1}(L)$ is the hyperbola whose equation is

$$
\frac{\left(x-\frac{y}{2 m}\right)^{2}}{\left(\frac{1}{2 m}+2 m\right) b}-\frac{y^{2}}{2 m b}=1 .
$$


Proof. Let $f\left(x_{0}+i y_{0}\right)=z=x+i y$, then $x=-2\left(x_{0}^{2}-y_{0}^{2}\right)$ and $y=$ $-4 x_{0} y_{0}$. Substitute into $y=m x+b$, simplify, and then replacing $x_{0}$ by $x$ and $y_{0}$ by $y$.

Recall that the line $L_{K}$ is the line with the equation $y=-\frac{\sqrt{3}}{3} x+2 \frac{\sqrt{3}}{3}$. We call this the $K$-line. The point of tangency of the $K$-line and the unit circle is $\left(\frac{1}{2}, \frac{\sqrt{3}}{2}\right)$.

Example 8.9. The preimage of the segment of the $K$-line between $x=\frac{1}{2}$ and $x=2$ is the portion of the hyperbola $4\left(x_{0}+\frac{\sqrt{3}}{2}\right)^{2}-7 y_{0}^{2}=3$ between $i \frac{\sqrt{2}}{2} e^{i \frac{\pi}{6}}$ and $-i$.

Example 8.10. Let $L_{\theta}$ denote the line through the origin making a counterclockwise angle of $\theta$ with the positive real axis. Then $f\left(L_{\theta}\right)=$ $L_{2 \theta}$.

Example 8.11. Recall that $\mathcal{A}_{1}$ is the region in the first quadrant bounded by the unit circle, the Schottky parabola, and $L_{\frac{\pi}{3}}$. Thus $f^{-1}\left(\mathcal{A}_{1}\right)$ will be bounded by the circle centered at the origin with radius $\frac{\sqrt{2}}{2}$, the line $x+y=1$ and $L_{\frac{\pi}{6}}$.

8.2. Second pre-image of the Schottky parabolas. We have seen that $f(\{x+i y \mid x \pm y= \pm 1\})$ is the Schottky parabolas. We want to describe $x_{0}+i y_{0}$, where $\left\{f\left(z_{0}\right)\right\}=\{x+i y \mid x \pm y= \pm 1\}$.

We note that $x=-2\left(x_{0}^{2}-y_{0}^{2}\right)$ and $y=-4 x_{0} y_{0}$. Thus

$$
-2\left(x_{0}^{2}-y_{0}^{2}\right) \pm\left(-4 x_{0} y_{0}\right)= \pm 1 \text {. }
$$

In particular,

$$
\frac{\left(x_{0} \mp y_{0}\right)^{2}}{ \pm \frac{1}{2}} \mp \frac{\left(y_{0}\right)^{2}}{\mp \frac{1}{4}}=1 .
$$

This proves

Corollary 8.12. The second inverse image of the Schottky parabolas consists of portions of eight hyperbolas. The hyperbolas intersect in pairs on points on the circle $C_{\frac{\sqrt{2}}{2}}=f^{-2}\left(C_{0}\right)$ and the portions of the hyperbolas between sucessive points of intesection gives the boundary of second classical T-Schottky space.

Figure 3 illustrates this. More generally, if $n \geq 2$, one expects $f^{-n}(\mathbf{P})$, the boundary of the $n$th classical space, to be the union of portions of branches of $2^{n+1}$ hyperbolas. These $2^{n+1}$ branches of hyperbolas intersect in pairs with the points of intersection lying on the 


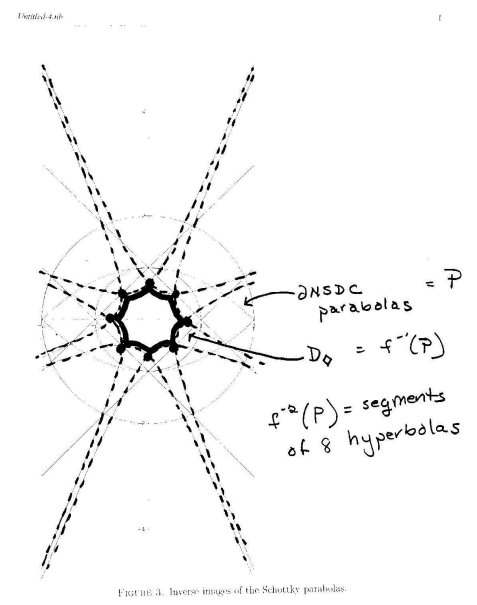

FiguRE 3. Inverse images of the Schottky parabolas.

circle $f^{-n}\left(C_{0}\right)$ which has radius $t_{n-1}=\frac{1}{2^{\frac{2^{n-1}-1}{2^{n-1}}}}$ and the boundary consists of the portions of the hyperbolas between successive intersection points. The $\left\{f^{-n}(\mathbf{P}), n \in \mathbb{Z}\right\}$ tessellate the complex plane.

Example 8.13. Loxodromic-Parabolic T-Schottky generators. Any given pair of generators of a classical Schottky or T-Schottky group may or may not be generators for an actual Schottky configuration, that is, the set of side pairings for two pairs of Schottky circles. If they are we say that they are Schottky generators for a marked Schottky configuration. In [7] it was shown that if a $G_{\lambda}$ was a T-Schottky group, it had a pair of parabolic Schottky generators for a marked Schottky configuration. It was also shown that $G_{\lambda}$ had an additional loxodromic-parabolic pair of Schottky generators for a marked Schottky configuration precisely when $\lambda=x+i y$ satisfied $x \pm y \geq \pm 2$ [7]. In particular if $D_{2 \diamond}$ is the square with this boundary its pre-image under $f$ consists of segments of the eight hyperbolas $\pm\left(x_{0} \mp y_{0}\right)^{2} \mp \frac{y_{0}^{2}}{\mp \frac{1}{2}}= \pm 1$ inside the unit circle that intersect at the $\pm 1, \pm i$, and $\frac{ \pm 1 \pm i}{2}$. See Figure 1.

\section{LAteral Iteration AND Vertical iteration}

We think of the iteration of subgroups given by 6.1 as being vertical iteration. The successive subgroups are moving down in the diagram of the lattice. We can also perform what we call a lateral iteration. That is, for each integer $n \neq 0, T_{n \lambda}=T_{\lambda}^{n}$ and we consider the subgroup $\left\langle S, T_{n \lambda}\right\rangle$ of $G_{\lambda}$. Now $\left\langle S, T_{\lambda}^{n}\right\rangle=\left\langle S, T_{n \lambda}\right\rangle=G_{n \lambda}$. 
We can also perform a single vertical iteration or a series of vertical iterations on $G_{n \lambda}$. A single vertical iteration replaces $G_{n \lambda}$ by $G_{-2 n \lambda^{2}}=$ $G_{-8 \lambda^{2}}$ and $m>0$, vertical iterations replaces $G_{n \lambda}$ by $G_{f^{m}(n \lambda)}$.

Since vertical iteration replaces $\lambda$ by $-2 \lambda^{2}$ and lateral iteration replaces $\lambda$ by $n \lambda$, we think of $V$ moving groups down in the lattice of subgroups more quickly than $L$. This motivates the names.

If we let $V\left(G_{\lambda}\right)=G_{\tilde{\lambda}}$ and $L^{n}\left(G_{\lambda}\right)=G_{n \lambda}$, then we can describe successive vertical and horizontal iterations by a sequence of $V^{m_{1}} L^{n_{1}} V^{m_{2}} \cdots L^{n_{t}}$ where $m_{1}, \ldots m_{t}$ and $n_{1}, \ldots, n_{t}$ are positive integers and $V^{m}$ denotes $m$ successive vertical iterations so that $V^{m}\left(G_{\lambda}\right)=G_{f^{m}(\lambda)}$.

Combining horizontal and vertical iteration allows one to move more fully around in the parameter space. We observe that

Proposition 9.1. (1) No applications of vertical iteration moves $\lambda$ out of the Jørgensen circle.

(2) There always is a lateral move that takes a given $\lambda$ out of the Jørgensen circle. That is, for each $\lambda \neq 0$ in the Jørgensen circle, there is a smallest $m$ such that $L^{m}\left(G_{\lambda}\right)=G_{m \lambda}$ lies outside the Jørgensen circle. That is, there is an integer $m$ such that $|m \lambda|>\frac{1}{2}$.

(3) $L$ and $V$ do not commute in general. That is if $n \neq 1$, $L^{n} \circ V\left(G_{\lambda}\right) \simeq G_{-2 n \lambda^{2}} \neq G_{-2 n^{2} \lambda^{2}} \simeq V \circ L^{n}\left(G_{\lambda}\right)$.

(4) However, there are some relations among the $L$ and $V$. For example

$$
V \circ L^{m}\left(G_{\lambda}\right)=L^{n} \circ V\left(G_{\lambda}\right) \Leftrightarrow n=m^{2} .
$$

One can use lateral iteration and obtain more formulas for parabolic dust and non-free groups.

Example 9.2. The NSDC parabolas under combined Vertical and Lateral iteration: In [7] non-separating circle groups, NSDC groups for short, were studied. It was found that the boundary of NSDC space is given by the portions of the two parabolas $y^{2}=16 \pm 8 x$ for $-2 \leq x \leq 2$. Let $S$ be the square with vertices at $\left( \pm \frac{1}{2}, \pm \frac{1}{2}\right)$. Then $V(S)$ consists of the parabolas $2 x= \pm 1+y^{2}$. Apply $L^{-2}$ (e.g. $z \mapsto-4 z$ ) to these parabolas to obtain the NSDC parabolas setting as setting $x=-\frac{x_{0}}{4}$ and $y=-\frac{y_{0}}{4}$ yields $y_{0}^{2}=16+8\left|x_{0}\right|$. That is, $L^{-2} \circ V(S)=\partial N S D C$. See Figure 1.

\section{The aCtion of $S$ And $T$ ON $\hat{\mathbb{C}}$.}

We have described the various types of groups by the regions in which $\lambda$ lies. We can equally well describe these groups by the configuration of Schottky curves imposed upon a group with a given type of $\lambda$. 


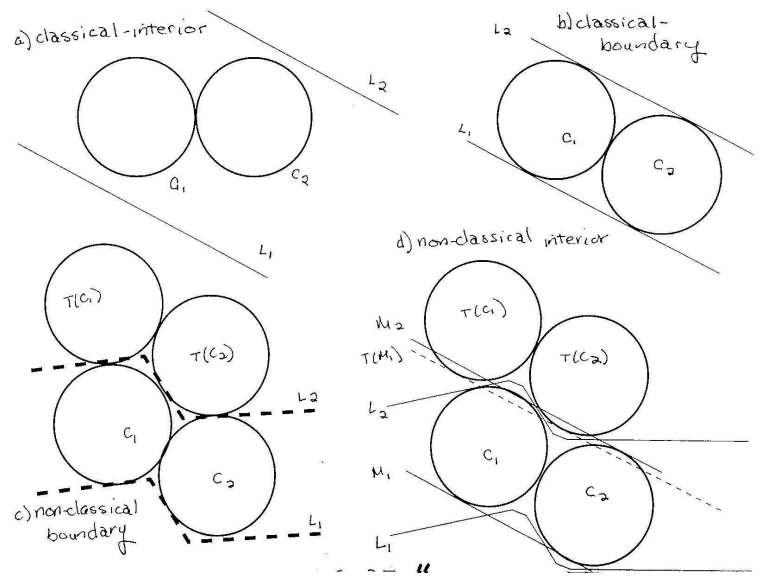

FiguRE 4. This figure illustrates the domains: a) the interior classical T-Schottky configuration, b) the boundary classical T-Schottky configuration, c) the interior non-classical configuration in the $\mathcal{N C F}$ family and $\mathrm{d}$ ) the non-classical boundary configuration for the $\mathcal{N C F}$ family.

If a rank two Schottky group has generators $g_{1}$ and $g_{2}$ and $g_{1}\left(C_{1}\right)=$ $C_{2}$ and $g_{2}\left(T_{1}\right)=T_{2}$ where $C_{1}, C_{2}, T_{1}$, and $T_{2}$ are Jordan curves whose interiors are pairwise disjoint, then the intersection of the exterior of these curves is called the Schottky domain and the set of circles together with the side pairings is called a Schottky configuration. If the Jordan curves are circles, we have a classical Schottky configuration. Not every set of generators for a Schottky group are the side pairings for a Schottky configuration. If $g_{1}$ and $g_{2}$ are the side pairings for a Schottky configuration, we call them the Schottky generators. In the case of $G_{\lambda}$ we normalized so that $C_{1}$ and $C_{2}$ paired by $S$ were are tangent at 0 with equal radii, $R$, and $T_{1}$ and $T_{2}$ paired by $T$ were tangent at $\infty$. We summarize the geometric configurations obtained for various regions. See Figure 4 .

Theorem 10.1. A $G_{\lambda}$ for $\lambda$ in the given region always has a Schottky configuration that can always be constructed as indicated.

$\lambda$ is an interior T-Schottky point: One can always find a classical configuration where the where the circles $C_{1}$ and $C_{2}$ lie interior to $T_{1}$ and $T_{2}$ and there are no additional tangencies between the circles. The configuration has a total of two points of tangency. 
$\lambda$ is $\partial$ T-Schottky: Every classical Schottky configuration will have six tangencies between the four circles except when $\lambda= \pm 2$ when there are only fours tangencies.

$\lambda$ lies on the lower boundary of $\mathcal{N C F}$, on the $K$-line: (or on the arc of the unit circle). The curves $C_{1}, C_{2}, T\left(C_{1}\right), T\left(C_{2}\right)$ will give a classical Schottky configuration for $\left\langle S, T S T^{-1}\right\rangle$. Here $C_{1}$ and $C_{2}$ are round circles. $T\left(C_{1}\right)$ is tangent to $C_{1}$ at a point $p_{1}$, $T\left(C_{2}\right)$ is tangent to $C_{2}$ at a point $p_{2}$, and $C_{2}$ is tangent to $T\left(C_{1}\right)$ at a point $p$. Let $M_{1}$ be the tangent line to $C_{1}$ through $p_{1}, M_{2}$ the tangent line to $C_{2}$ through $p_{2} ;$ and $M_{3}$ the tangent line to $T\left(C_{1}\right)$ and $C_{2}$ through $p$. Let $L_{2}$ be the Jordan curve made up of three arcs: the segment of $M_{1}$ between $\infty$ and $M_{1} \cap M_{3}$, the segment of $M_{3}$ between $M_{1} \cap M_{3}$ and $M_{3} \cap M_{2}$, and the segment $M_{2}$ between $M_{3} \cap M_{2}$ and $\infty$. Set $L_{1}=T^{-1}\left(L_{2}\right)$. Then the transformation $T$ pairs the Jordan curves $L_{1}$ and $L_{2}$.

$\lambda$ interior to $\mathcal{N C F}$ : Let $M_{1}$ and $M_{2}$ be parallel lines with each tangent to both $C_{1}$ and $C_{2}$. We assume that $T\left(M_{1}\right)$ lies between $M_{1}$ and $M_{2}$. Let $P$ be the perpendicular bisector of the line connecting the centers of $C_{1}$ and $C_{2}$. Let $M$ be a line parallel to $M_{1}$ that intersects $P$ at $q_{0}$, a point between the point of tangency of the $C_{1}$ and $C_{2}$ and $M_{2} \cap P$. Let $q$ be the point on $P$ a distance $R$ from $q_{0}$. Let $2 \lambda$ be the vector from 0 to $q$ and let $T(z)=$ $z+2 \lambda$. Let $L_{2}$ be the Jordan curve comprised of arcs along the perpendicular bisector of the centers of $C_{1}$ and $T\left(C_{1}\right)$, along $M$, along the perpendicular bisector of the centers of $T\left(C_{1}\right)$ and $C_{2}$, along the line parallel to $M$ equidistant from $M_{2}$, and along the perpendicular bisector of the line segment connecting the centers of $C_{2}$ and $T\left(C_{2}\right)$. Let $L_{1}=T^{-1}\left(L_{2}\right)$. Then $L_{1}, L_{2}=T\left(L_{1}\right), C_{1}$ and $C_{2}=S\left(C_{1}\right)$ give a Schottky domain for $G_{\lambda}$ and $\lambda$ will be interior to $\mathcal{N C F}$.

Proof. The description for the first three regions come from [7]. The description for the last follows by ruling out the configurations for first three cases.

\section{TOWARDS A MORE GENERAL THEORY}

If one looks in the bigger space of representations modulo conjugacy for two generator groups, what we have termed lateral is really semi-lateral iteration, $S L$. It is lateral only in comparison with $V$. A general Nielsen transformation sends generators $(A, B)$ to generators $\left(A, A^{ \pm 1} B\right)$, does not change the group, and does not change the properties of discreteness or non-discreteness or free-ness. This is true 
lateral iteration. It does change the triple of parameters one associates to a two generator group. We note that in the case of a discrete group, such a Nielsen transformation, $N$, corresponds to an element of the mapping-class group and thus has a geometric interpretation as a Dehn twist.

In our case, the Nieslen transformation moves us out of the twoparabolic parameter space. But when we move to a more general theory, it might be that certain sequences of moves in the larger parameter space bring us back to the two-parabolic space. We would like to develop a theory of motions around the representation space using the three types, $V, S L$ and $N$. This would be related to the various word families studied in connection with representations and volumes of hyperbolic manifolds and orbifolds.

\section{ACKNowledgements}

The author thanks David Wright for a number of helpful e-mail exchanges. The author also thanks Yair Minsky and the Yale Mathematics Department for their hospitality and support while some of this work was carried out.

\section{REFERENCES}

[1] Agol, Ian, Classification of non-free 2-parabolic generator Kleinian groups, Austin AMS mtg., Budapest Bolyai conference. Notes at http://www2.math.uic.edu/ agol/parabolic/parabolic01.html.

[2] Bamberg, J. Non-free points in groups generated by a pair of $2 \times 2$ matrices,

[3] Beardon, A. Some remarks on non-discrete Möbius groups Ann. Acad. Sci. Fenn, 21 (1996) 69-79.

[4] , Pell's equation and two generator free Möbius Groups, Bull. London Math. Soc. 25 (1993), no. 6, 527-532.

[5] Chang,B, Jennings, S.A. and Ree, R, On certains pairs of matrices which generate free groups, Canad. J. Math 10 (1958), 279-284.

[6] Gehring, F. W., Machlachlan, C., and Martin G.J, Two-generator Arithmetic Kleinian groups, II, Bull. London Math Soc. 30 (1998) 258-266.

[7] Gilman, J. and Waterman, P. Classical T-Schottky groups, J. D'Analyse (2006). bibitemJsum Gilman, J. Boundaries for two parabolic Schottky groups Spaces of Kleinian Groups, LMS Lecture Notes 329, Cambridge U. Press (2004) 283-299.

[8] Ignativ, Ju. A, Rational non-free points in the complex plane in Algorithmic problems in the theory of groups and semigroups, Tulsk. Gos. Ped. Inst., Tula 127 (1986), 72-80.

[9] Ignativ, Ju. A, Rational non-free points in the complex plane, II in Algorithmic problems in the theory of groups and semigroups, Tulsk. Gos. Ped. Inst., Tula (1990), 53-59.

[10] Keen, Linda and Series, Caroline The Riley Slice of Schottky Space, 
[11] Lyndon and Ullman, Groups generated by two parabolic fractional linear transformation, Canad. J. Math 21 (1969), 1388-1403.

[12] Marden, A. geometrically Finite Kleinian Groups and their Deformation Spaces in Discontinuous Groups and Automorphic Functions, ed. W.J. Harvey, Academic Press (1977) 261-293.

[13] Maskit, B. Kleinian Groups, Springer-Verlag (1988).

[14] Mumford, D. , Series, C and Wright, D, Indra's Pearls, (2002) Cambridge University Press.

[15] Ratcliffe, J. Foundations of Hyperbolic Manifolds, Springer-Verlag (1994).

[16] Ree, R. On certain pairs of matrices which do not generate a free group Canad. Math. Bulletin 4, (1961), 49-52.

[17] , Wright, David J., unpublished ( 1980).

[18] Wright, David J., Searching for the cusp, Spaces of Kleinian Groups, LMS Lecture Notes 329, Cambridge U. Press (2004), 1-36.

Mathematics Department, Rutgers University, Newark, NJ 07102 e-mail: gilman@andromeda.rutgers.edu 